Toward highly conductive $n$-type diamond: Incremental phosphorus-donor concentrations assisted by surface migration of admolecules

Takashi Yamamoto', Stoffel D. Janssens, Ryota Ohtani, Daisuke Takeuchi, and Satoshi Koizumi'

Citation: Appl. Phys. Lett. 109, 182102 (2016); doi: 10.1063/1.4966287

View online: http://dx.doi.org/10.1063/1.4966287

View Table of Contents: http://aip.scitation.org/toc/apl/109/18

Published by the American Institute of Physics 


\title{
Toward highly conductive $n$-type diamond: Incremental phosphorus-donor concentrations assisted by surface migration of admolecules
}

\author{
Takashi Yamamoto, ${ }^{1,2, a)}$ Stoffel D. Janssens, ${ }^{1}$ Ryota Ohtani, ${ }^{1}$ Daisuke Takeuchi, ${ }^{2,3}$ \\ and Satoshi Koizumi ${ }^{1,2, b)}$ \\ ${ }^{1}$ National Institute for Materials Science (NIMS), 1-1 Namiki, 305-0044 Tsukuba, Japan \\ ${ }^{2}$ Advanced Low Carbon Technology Research and Development Program (ALCA), Japan Science and \\ Technology Agency (JST), clo AIST, 1-1-1 Umezono, 305-8568 Tsukuba, Japan \\ ${ }^{3}$ National Institute of Advanced Industrial Science and Technology (AIST), 1-1-1 Umezono, 305-8568 Tsukuba, \\ Japan
}

(Received 24 July 2016; accepted 13 October 2016; published online 31 October 2016)

\begin{abstract}
The realization of low-resistance $n$-type diamond is required to form novel semiconducting devices. However, heavily doping with phosphorous, the most suitable electron donor, remains challenging. Here we demonstrate that the phosphorus incorporation efficiency in deposited diamond can be maximized when using the largest possible terrace width of vicinal $\{111\}$-substrates. Given step-flow-predominant crystal growth, the greater surface migration length of phosphoruscontaining admolecules compared with those of carbon-containing parent species explain this. With our findings we create a model which provides a complementary perspective to explain large fluctuations in dopant incorporation efficiencies for $p$-type and $n$-type diamond. Our model can also explain conflicting models for admolecule motion responsible for diamond crystallization. Published by AIP Publishing. [http://dx.doi.org/10.1063/1.4966287]
\end{abstract}

The most-advanced semiconducting diamond applications in power electronics ${ }^{1}$ and optoelectronics ${ }^{2}$ under severe environments in addition to quantum communication and processing at room temperature, ${ }^{3}$ capitalizing on "adamant" properties of diamond, have arisen from the discovery of synthetic $n$-type diamond. ${ }^{4}$ Considerable progress has been made in heavily doping phosphorus donors, ${ }^{5,6}$ with a strategy shift to make diamond more conductive, particularly, by using a hopping conduction ${ }^{7}$ that emerges above $[\mathrm{P}] /[\mathrm{C}]>10^{20} \mathrm{~cm}^{3}$; however, there remains the challenge to achieve such high solubility.

Diamond crystallization from gas phase occurs by a unique nonequilibrium chemical reaction. ${ }^{8-10}$ Ongoing studies of chemical vapor deposition (CVD) diamond growth since the pioneering work performed in the $1970 \mathrm{~s}^{11}$ and $1980 \mathrm{~s}^{12}$ have led to the most evident fact that atomic hydrogen plays a key role in stabilizing the diamond phase while reducing other phases, including graphite and amorphous/diamond-like carbon. ${ }^{10}$ Therefore, diamond crystallizes under the limiting conditions of an extremely diluted source gas of hydrocarbon with hydrogen (typically, $\left[\mathrm{CH}_{4}\right] /\left[\mathrm{H}_{2}\right]=0.1 \%-3 \%$ in gas-phase volume) and specific growth temperatures of $800-1100^{\circ} \mathrm{C}$ (ca. $50 \%$ of the Debye temperature of diamond). Mass spectroscopy and kinetics considerations have mutually indicated the principal gaseous precursors as being $\mathrm{CH}_{3}$ radicals among possible hydrocarbon fragments $\left(\mathrm{CH}_{x}\right.$ with $x=0-4$ or $\mathrm{C}_{2} \mathrm{H}_{y}$ with $y=1-6)$-also, $\mathrm{C}_{2} \mathrm{H}_{2}$ is possible-and inferred the chemisorption of them to $s p^{3}$-hybridized surface radical sites (dangling bonds) followed by the formation and migration of $\mathrm{CH}_{2}$-species on the surface by hydrogen abstraction reactions. ${ }^{13-15}$

\footnotetext{
${ }^{a)}$ Present address: Institute for Materials Research (IMO), Hasselt University, Wetenschapspark 1, B-3590 Diepenbeek, Belgium. Electronic mail: takashi. yamamoto@uhasselt.be

b)KOIZUMI.Satoshi@nims.go.jp
}

However, it should be noted that the role of surface migration remains controversial. ${ }^{16,17}$

In the present study, we assessed admolecule motion, applying the Burton-Cabrera-Frank theory, ${ }^{18}$ by using a simple method of manipulating crystal growth kinetics, which comprised epitaxy on vicinal faces tilted from an idiomorphic crystal surface. From our model, admolecule motion might predominantly limit phosphorus incorporation. ${ }^{5,6} \mathrm{We}$ also provide a complementary perspective to explain different dopant incorporation in $p$-type ${ }^{19}$ and $n$-type diamonds to the changes in misorientation angle, and for the admolecule motion ${ }^{13-17}$ that is presently poorly understood.

Phosphorus-doped homoepitaxial diamond films were grown on two vicinal $\{111\}$-diamond substrates (hereafter referred to as sample 1 and sample 2) by microwave plasmaassisted chemical vapor deposition, ${ }^{20}$ using a self-designed reactor. $^{21}$ The substrate used was a high-pressure high-temperature synthesized $\{111\}$ type Ib diamond (Sumitomo co.) of $2 \times 2 \mathrm{~mm}^{2}$ surface and $0.5 \mathrm{~mm}$ thickness after laser-cutting along $[\overline{1} 2 \overline{1}]$ and $[10 \overline{1}]$. Both surfaces were mirror polished to be parallel to the (111) crystal plane with a misorientation angle, $\theta_{\text {mis }}$, of $\sim 5.5^{\circ}$ (SYNTEK co., Ltd.) and then different misorientation angles of $-0.5^{\circ} \leq \theta_{\text {mis }} \leq 5.8^{\circ}$ towards a $[\overline{1} 2 \overline{1}]$ misorientation vector $\boldsymbol{a}_{\text {mis }}$ at plus $\theta_{\text {mis }}$ or [12 1$]$ at minus $\theta_{\text {mis }}$, defined as shown in Figs. 1(a) and 1(b), were provided on one surface of the substrate by mechanical polishing, resulting in a wedge shape on the lateral face (Fig. 1(b)). The misorientation angles were determined with ca. $0.2^{\circ}$ accuracy by combination of x-ray diffraction and laser microscopy.

In microwave plasma assisted CVD, thin films were grown by a mixture of hydrogen $\left(\mathrm{H}_{2}\right.$, a purity of $\left.9 \mathrm{~N}\right)$, methane $\left(\mathrm{CH}_{4}, 6 \mathrm{~N}\right)$, and phosphine diluted with hydrogen $\left(\mathrm{PH}_{3} / \mathrm{H}_{2}\right.$ $=1000 \mathrm{ppm}, 6 \mathrm{~N})$. The total gas flow rate and pressure were $1000 \mathrm{sccm}$ and 100 Torr $\left(=1.34 \times 10^{4} \mathrm{~Pa}\right)$, respectively. The concentration ratios of $\left[\mathrm{CH}_{4}\right] /\left[\mathrm{H}_{2}\right]$ and $\left[\mathrm{PH}_{3}\right] /\left[\mathrm{CH}_{4}\right]$ 
(a)

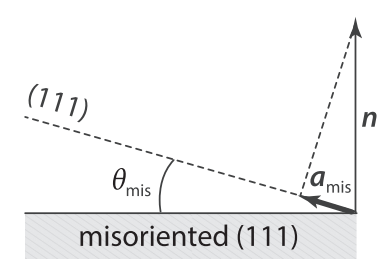

(b)

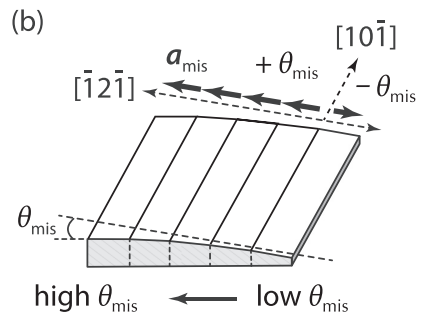

(c)

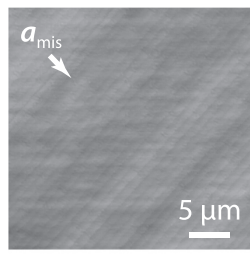

$\theta_{\text {mis }}:-0.5^{\circ}, R_{\mathrm{a}}: 5 \mathrm{~nm}$ $T_{\mathrm{f}}: 0.30 \mu \mathrm{m}$

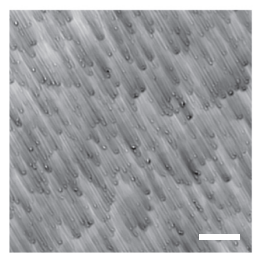

$2.4^{\circ}, 12 \mathrm{~nm}, 1.32 \mu \mathrm{m}$

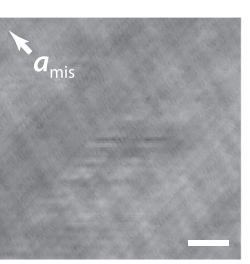

$0.3^{\circ}, 6 \mathrm{~nm}, 0.37 \mu \mathrm{m}$

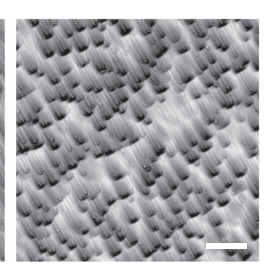

$3.4^{\circ}, 21 \mathrm{~nm}, 1.31 \mu \mathrm{m} 5.5^{\circ}, 34 \mathrm{~nm}, 1.40 \mu \mathrm{m}$

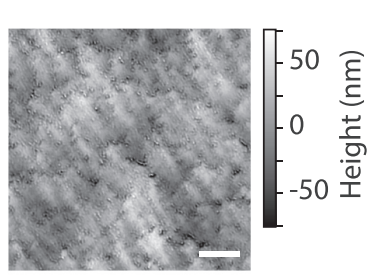

$1.4^{\circ}, 19 \mathrm{~nm}, 0.83 \mu \mathrm{m}$

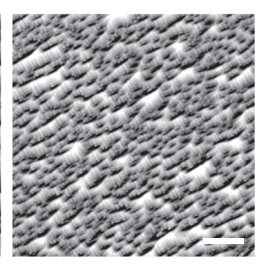

(d)

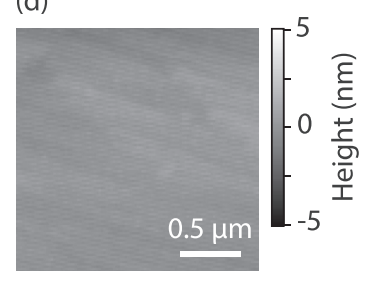

$R_{\mathrm{a}}: 0.2 \mathrm{~nm}, T_{\mathrm{f}}<0.3 \mu \mathrm{m}$

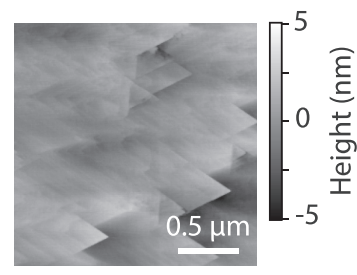

$R_{\mathrm{a}}: 0.7 \mathrm{~nm}, T_{\mathrm{f}}=0.3 \mu \mathrm{m}$

FIG. 1. (a) Misorientation angle $\left(\theta_{\text {mis }}\right)$ defined as the misalignment angle between the polished substrate surface and the (111)-plane. The miosrientation vector $\left(\boldsymbol{a}_{\mathrm{mis}}\right)$ presents how misoriented planes tilt toward either crystal axis that lies in the (111)-plane, where $\boldsymbol{a}_{\text {mis }}$ is the projection vector of $\boldsymbol{n}$ onto (111) and $\boldsymbol{n}$ the normal vector to the misoriented plane. (b) Schematic of the laser-cut substrate along two orthogonal directions of [1 $2 \overline{1}]$ and $[10 \overline{1}]$, with various $\theta_{\text {mis }}$ $\left(-0.5^{\circ} \leq \theta_{\text {mis }} \leq 5.8^{\circ}\right)$ on the one surface of the substrate, which leads to a wedge-shaped side face. (c) Laser-microscope images of growth surfaces for various misorientation angles $\left(\theta_{\mathrm{mis}}=-0.5^{\circ}\right.$ to $\left.5.5^{\circ}\right)$ and film thicknesses $\left(T_{\mathrm{f}}=0.3-1.4 \mu \mathrm{m}\right)$ with 0.15 - $\mu \mathrm{m}$ height scale. $R_{\mathrm{a}}$ is the arithmetic mean roughness. The misorientation directions $\left(\boldsymbol{a}_{\mathrm{mis}}\right)$ for $\theta_{\mathrm{mis}} \geq 1.4^{\circ}$ are the same as that of $0.3^{\circ}$. The height zero in the height scale corresponds to the mean height of each growth surface. Note that the striped lines perpendicular to $\boldsymbol{a}_{\text {mis }}$ at $\left|\theta_{\text {mis }}\right| \leq 0.5^{\circ}$ are not caused by step bunching but interference of the light because of the wedge shape of the substrate (as shown in (b)). The observed fringe intervals are well described by the diffraction condition of $(m+1 / 2) \frac{\lambda}{2 n \tan \theta}$, where $m$ is the integer number $(m \geq 0), n$ the refractive index of diamond, $\theta$ the wedge angle as $5.5^{\circ}-\theta_{\text {mis }}$, and $\lambda$ the laser wavelength of 408 nm. (d) Atomic-force-microscopy images of two as-grown surfaces over a $2 \times 2 \mu \mathrm{m}^{2}$ area with $10 \mathrm{~nm}$ height scale: (top figure) $T_{\mathrm{f}}<0.3 \mu \mathrm{m}$ and (bottom) $T_{\mathrm{f}} \sim 0.3 \mu \mathrm{m}$.

were adjusted through mass flow controllers to $0.05 \%$ and $1 \%$, respectively. Growth was performed during $2 \mathrm{~h}$ with a microwave input power of ca. $310 \mathrm{~W}$ which lead to a substrate temperature of ca. $930^{\circ} \mathrm{C}$ monitored by a radiation thermometer. We have optimized our recipe for step-flow growth $\left(\left[\mathrm{CH}_{4}\right] /\left[\mathrm{H}_{2}\right]=0.05 \%\right.$ with $\left[\mathrm{PH}_{3}\right] /\left[\mathrm{CH}_{4}\right]<10^{3} \mathrm{ppm}$ (Ref. 22)), currently up to a 1.2- $\mu$ m film thickness for which no two-dimensional nucleation occurs (see Refs. 4, and 23-25 for information on crystallographic characterization). In the present study, an increased gaseous phosphorus concentration of $10^{4} \mathrm{ppm}$ was used to compare our results with leading-edge results ${ }^{5,6}$ obtained for highly phosphorus doped diamond (ca. $10^{20} \mathrm{~cm}^{-3}$ in the solid phase).

Phosphorus concentrations in the deposited films were determined from secondary ion mass spectroscopy (SIMS) depth profiles across the film-substrate interface, which also allowed measurement of film thickness. The growth rate $R_{\mathrm{g}}$ with respect to $\theta_{\text {mis }}$ was estimated by dividing the measured film thickness by the growth time. Our previous studies have confirmed that a height difference up to ca. $0.2 \mathrm{~mm}$ between the top surface of the substrates and the lower end of the plasma ball (see Ref. 21 for information on our sample holder) leads to non-detectable changes in $C_{\mathrm{p}}$ and $R_{\mathrm{g}}$. Therefore, we assume that the different separation distance for each $\theta_{\text {mis }}$ resulting from the wedge-shaped substrate (Fig. 1(b)) should contribute minimally to both $C_{\mathrm{p}}$ and $R_{\mathrm{g}}$ in the present study.

Figure 1(c) presents images obtained with a threedimensional laser scanning microscope of the as-grown surface over a $30 \times 30 \mu \mathrm{m}^{2}$ areas. The arithmetic mean roughness is denoted by $R_{\mathrm{a}}$. Surface tended to be smoother and smoother with decreasing $\left|\theta_{\text {mis }}\right|$ except at $2.4^{\circ}$. Two flat surfaces below ca. $10 \mathrm{~nm}$ resolution in laser microscopy were obtained when the film thickness $\left(T_{\mathrm{f}}\right)$ was thiner than $0.4 \mu \mathrm{m}$ (see the data taken at $\left|\theta_{\text {mis }}\right| \leq 0.5^{\circ}$ ). When the $T_{\mathrm{f}}$ increased further at $>1.4^{\circ}$, the evolution of dislocation growth, resulting in deformed triangular pyramids which overlap each other, became observable by laser microscopy. We attribute the pyramids to a screw dislocation growth resulting from phosphorus-induced lattice strain accumulation since the covalent radius of phosphorus $(1.10 \AA)$ is much larger than that of carbon $(0.77 \AA)$. The different $T_{\mathrm{f}}$ originated from the incremental growth rate with increasing $\theta_{\text {mis }}$ - as we demonstrate later. In addition, atomic force microscopy revealed that the surfaces at $\left|\theta_{\text {mis }}\right| \leq 0.5^{\circ}$ were very smooth $\left(R_{\mathrm{a}}=0.2 \mathrm{~nm}\right)$ when $T_{\mathrm{f}}<0.3 \mu \mathrm{m}$, while triangular pyramids started to appear when $T_{\mathrm{f}} \geq 0.3 \mu \mathrm{m}$, as shown in Fig. 1(d).

Figure 2(a) represents the depth profiles of phosphorus incorporation concentration $C_{\mathrm{p}}$ in the diamond lattice for various misorientation angles (sample 1) by secondary ion mass spectroscopy (SIMS), with $C_{\mathrm{p}}=[\mathrm{P}] /[\mathrm{C}]$. The SIMS measurements across the film-substrate interface allowed the discrimination of $\mathrm{Ib}$ substrates (illustrated by the gray-shade area) from phosphorus-doped films. The $C_{\mathrm{p}}$ at $\left|\theta_{\text {mis }}\right| \leq 0.5^{\circ}$ remains unchanged over the entire range of film thickness, whereas $C_{\mathrm{p}}$ at $\left|\theta_{\text {mis }}\right| \geq 2^{\circ}$ gradually increased with the increasing of the film thickness. Figure 2(b) shows $C_{\mathrm{p}}$ as a function of average terrace width, $w=d / \tan \theta_{\text {mis }}$ ( $d$ is the theoretical step height on $\{111\}$ surface, $2.06 \AA$ ). Here we use the nominal $w$ since the actual terrace width was not possible to be estimated by atomic force microscopy. We also plot the early-stage $C_{\mathrm{p}}$ values taken at a $0.25 \mu \mathrm{m}$-depth of each SIMS profile in order to neglect the effect of the evolution of phosphorus-induced dislocation growth on $C_{\mathrm{p}}$. We found that the $C_{\mathrm{p}}$ increased with expanding $w$ and thus maximum values of $9.5 \times 10^{19} \mathrm{~cm}^{-3}$ (sample 1) and $7.9 \times 10^{19} \mathrm{~cm}^{-3}$ (sample 2) were obtained at the smallest $\theta_{\text {mis }}$ (Fig. 2(b)). Dividing $C_{\mathrm{p}}$ by the phosphorus source gas concentration gives the incorporation efficiency $([\mathrm{P}] /[\mathrm{C}]) /\left(\left[\mathrm{PH}_{3}\right] /\left[\mathrm{CH}_{4}\right]\right)=5.4 \%$ at $\left|\theta_{\text {mis }}\right|=0.35^{\circ}$ (sample 
(a)

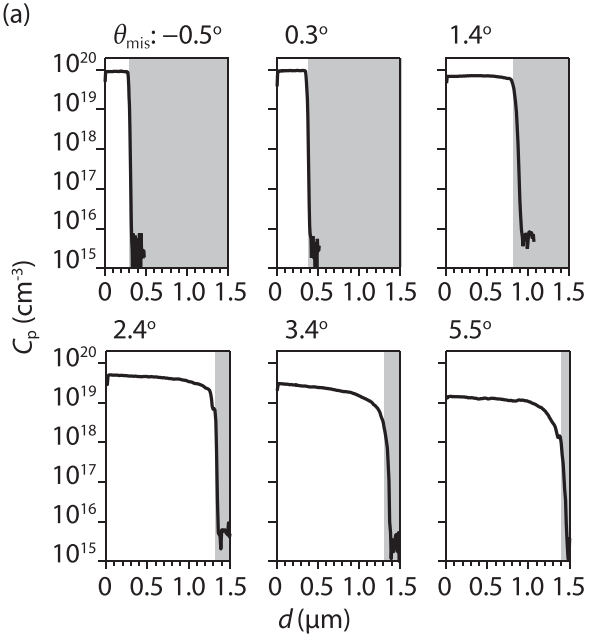

(b)

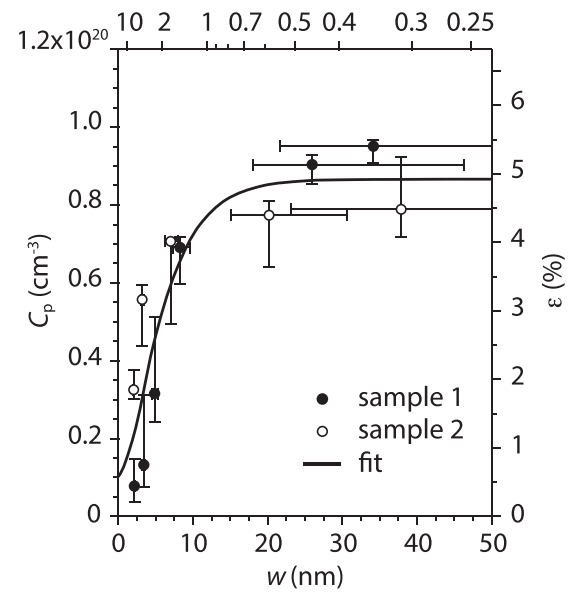

(c)

$\theta_{\text {mis }}\left(^{\circ}\right)$

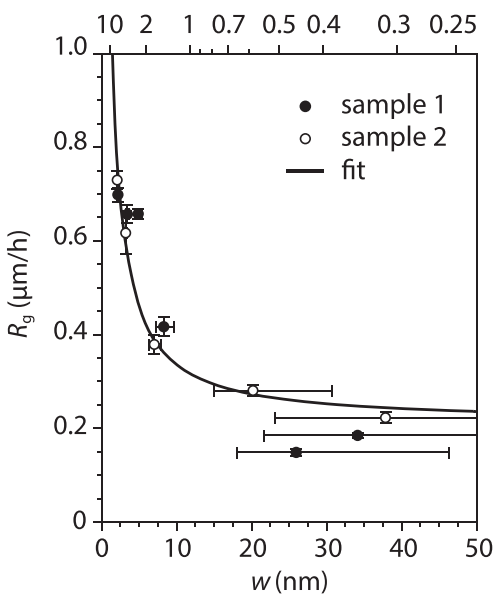

FIG. 2. (a) Depth profiles of phosphorus incorporation concentrations $\left(C_{\mathrm{p}}\right.$, solid lines) on semi-logarithmic scale for various misorientation angles (sample 1 ) by secondary ion mass spectroscopy (SIMS), where Ib substrates are shown by the gray shade. (b) $C_{\mathrm{p}}$ (left axis) and the corresponding phosphorusincorporation efficiency from the gas phase $\epsilon$ (right axis) with respect to the terrace width $w=d / \tan \theta_{\text {mis }}(d=2.06 \AA$ ). The data for sample 1 (solid circles) and sample 2 (open circles) are plotted with the fitting curve (solid line). Each data point represents the early-stage $C_{\mathrm{p}}$ value taken at a $0.25 \mu \mathrm{m}$-depth of SIMS data and the error bars show the maximum and minimum values. The lateral error bars indicate the accuracy of $\theta_{\text {mis }}-$ measurement. (c) Growth rate $R_{\mathrm{g}}$ of phosphorus-doped diamonds as a function of $w$ (solid and open circles) with the curve fitting (solid line), where the uncertainty for the determination of the film-substrate interface of each SIMS depth profile is shown as the error bar. The lateral error bars correspond to the accuracy of $\theta_{\text {mis }}$-measurement.

1) and $4.5 \%$ at $\left|\theta_{\text {mis }}\right|=0.31^{\circ}$ (sample 2 ), as shown in the right axis of Fig. 2(b). The efficiency at the minimal $\left|\theta_{\text {mis }}\right|$ is 12.2 (sample 1) and 2.4 (sample 2) times higher than that at $\theta_{\text {mis }}>$ $5.5^{\circ}$ and improved by an order of magnitude compared with previous studies ${ }^{5,6}$ where similar $C_{\mathrm{p}}$ values were obtained. The reason for the considerable difference in $C_{\mathrm{p}}$ between the two samples is unclear at present. In contrast to the tendency of $C_{\mathrm{p}}$, the growth rate $R_{\mathrm{g}}$ decreases with increasing $w$ (Fig. 2(c)).

We formulate an analytical model (see supplementary material) based on the Burton-Cabrera-Frank theory ${ }^{18}$ as

$$
C_{\mathrm{p}}=\alpha \frac{\tilde{x}_{\mathrm{s}} \tanh \left(w / 2 \tilde{x}_{\mathrm{s}}\right)}{x_{\mathrm{s}} \tanh \left(w / 2 x_{\mathrm{s}}\right)},
$$

where $\alpha$ is the constant term determined by growth conditions and materials, and $\tilde{x}_{\mathrm{s}}$ and $x_{\mathrm{s}}$ the mean migration length of phosphorus-containing admolecules (including $\mathrm{PH}_{x}$ and, probably, $\mathrm{CPH}_{y}$, hereafter referred to as phosphorus admolecules) and hydrocarbon admolecules (including $\mathrm{CH}_{x}$ and $\mathrm{C}_{2} \mathrm{H}_{y}$ ), respectively, and $w$ the calculated terrace width. By fitting the data using Eq. (1) (the solid line in Fig. 2(b)), we obtain $x_{\mathrm{s}}=0.49 \mathrm{~nm}$, indicating that hydrocarbon admolecules migrated for only a distance comparable to the diamond lattice constant (ca. $0.36 \mathrm{~nm}$ ). For phosphorus, the migration length is remarkably longer $\left(\tilde{x}_{\mathrm{s}}=4.16 \mathrm{~nm}, \tilde{x}_{\mathrm{s}} / x_{\mathrm{s}}=8.45\right)$. Putting the obtained $x_{\mathrm{s}}$ into the following equation as

$$
R_{\mathrm{g}}=\gamma \frac{x_{\mathrm{s}}}{w} \tanh \left(\frac{w}{2 x_{\mathrm{s}}}\right)+R_{0},
$$

the growth rate $R_{\mathrm{g}}$ can be explained by this model (solid line of Fig. 2(c)). Here $\gamma$ is the rate constant determined by the growth conditions and chemical reactions on the surface related to diamond growth mechanism itself, and $R_{0}$ obtained as being $0.21 \mu \mathrm{m} / \mathrm{h}$ is the $\theta_{\text {mis }}$-independent component related to phosphorus-induced dislocation growth.
Figure 3 shows the schematic of the incorporation of phosphorus atoms (black cube) for each flowing step (gray) for a high $\theta_{\text {mis }}$ (top figure) and a low $\theta_{\text {mis }}$ (bottom). When $w$ becomes larger, the incorporation-the reachability into step edges — of hydrocarbon admolecules decreases more rapidly than that of phosphorus admolecules due to $x_{\mathrm{s}} / \tilde{x}_{\mathrm{s}}<1$. Therefore, $C_{\mathrm{p}}$ increases with expanding $w$ and finally reaches a maximum level near $2 \tilde{x} / w=1$ (as described in Eq. (1)).

Figure 4 shows a scaling of dopant concentrations $(C)$ with respect to the terrace width $(w)$ for various values of $\tilde{x}_{\mathrm{s}} / x_{\mathrm{s}}$ (indicated in the figure), with $C_{w \rightarrow 0}$ the dopant concentration for $w \rightarrow 0$ and with $x_{\mathrm{s}}^{l}$ either $\tilde{x}_{\mathrm{s}}$ or $x_{\mathrm{s}}$, depending on
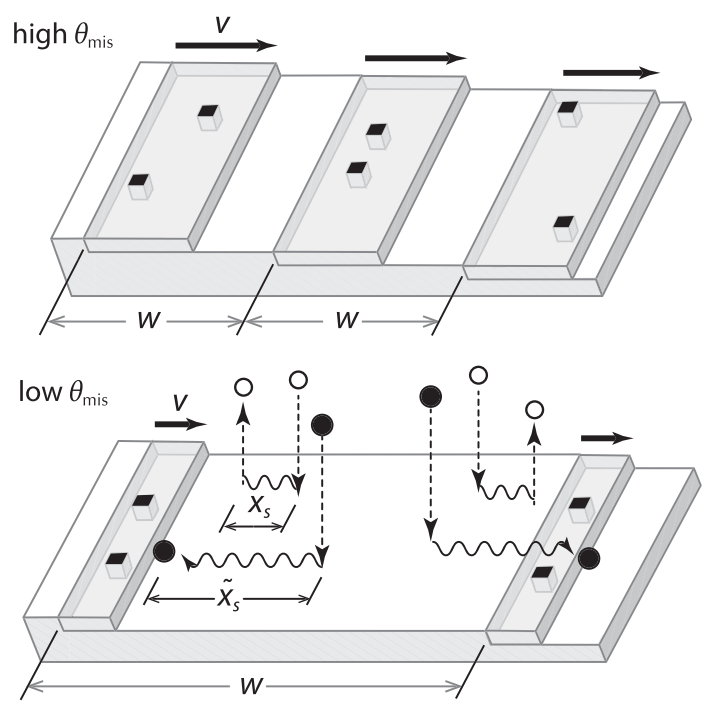

FIG. 3. Schematics of an incorporation of phosphorus atoms (black cubes) for each flowing steps (gray) with a velocity of step advance $(v)$ for a high $\theta_{\text {mis }}$ (top figure) and a low $\theta_{\text {mis }}$ (bottom figure). A longer surface migration length $\left(\tilde{x}_{\mathrm{s}}\right)$ of phosphorus admolecules (solid circles) than that $\left(x_{\mathrm{s}}\right)$ of hydrocarbon admolecules results in a higher surface concentration of phosphorus donors when expanding the terrace width $w$. 


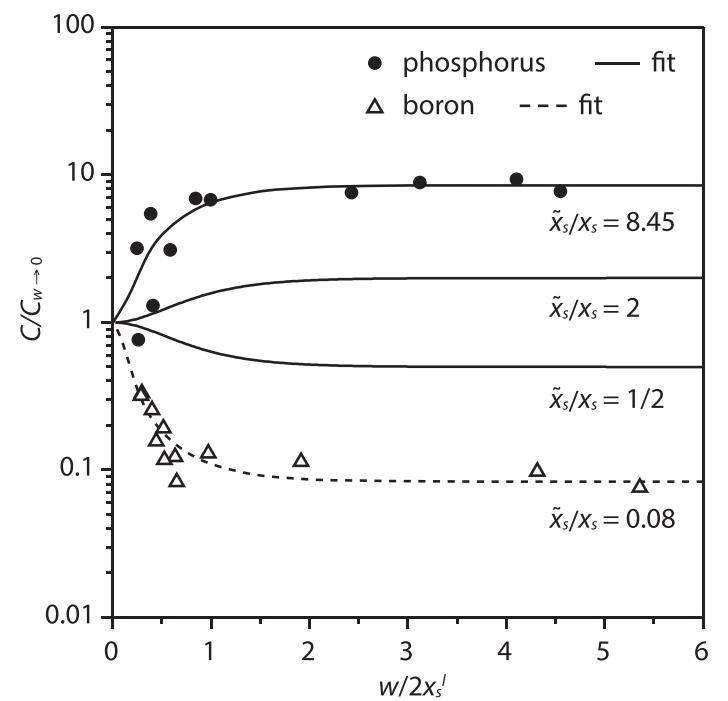

FIG. 4. Normalized dopant concentrations $C / C_{w \rightarrow 0}$ as a function of normalized terrace width $w / 2 x_{\mathrm{s}}^{l}$ for different values of $\tilde{x}_{\mathrm{s}} / x_{\mathrm{s}}$, where $C_{w \rightarrow 0}$ is the dopant concentration at the terrace width $w \rightarrow 0, \tilde{x}_{\mathrm{s}}$ and $x_{\mathrm{s}}$ are the mean migration lengths of admolecules for dopant/impurity- and parent-species, respectively, and $x_{\mathrm{s}}^{l}$ is either $\tilde{x}_{\mathrm{s}}$ or $x_{\mathrm{s}}$, depending on which one is larger. The experimental data of phosphorus-doped $\{111\}$-diamond (solid circles) with the fit curve (solid line) is taken from Fig. 2(b). The result of boron-doped $\{100\}$-diamond (open triangles) is quoted from Ref. 19 and then analyzed here as shown in the fit curve (dashed line).

which one is longer. The trend observed for the phosphorus concentration (solid circles in Fig. 4) is opposite to that of the boron concentration on misoriented $\{100\}$ surfaces reported previously. ${ }^{19}$ We attribute this to a shorter migration length of boron-admolecules than that of hydrocarbonadmolecules, that is, a ratio of $\tilde{x}_{\mathrm{s}} / x_{\mathrm{s}}=0.08\left(\tilde{x}_{\mathrm{s}}=0.14 \mathrm{~nm}\right.$ and $x_{\mathrm{s}}=1.70 \mathrm{~nm}$ ) by plotting the data (open triangles) with the fit curve (dashed line). The scaling would be universal for various dopant (or impurity) incorporation in different systems, and thus, $C / C_{w \rightarrow 0}$ at $w \rightarrow 0$ changes by a factor of a $\tilde{x}_{\mathrm{s}} / x_{\mathrm{s}}$ when $w \rightarrow \infty$.

Many studies have argued that hydrocarbon admolecules are unlikely to migrate on diamond surfaces due more probably to their strong bonding to the surface ${ }^{26}$ or Eley-Rideal type reactions (the chemisorption of admolecules onto the terrace of the diamond surface followed by the etching ${ }^{17}$ ). Yet in some theoretical considerations, it was proposed that the transport of a covalently bonded group $-\mathrm{CH}_{2}$ bridging on a dimer for $\{100\}-(2 \times 1)$ :H surfaces-in a manner equivalent to surface migration might occur in the presence of hydrogen. ${ }^{13,14}$ This migration length was estimated to be ca. $10 \AA{ }^{14}$ which reasonably agrees with that of ca. $17 \AA$ obtained from the data of $p$-type $\{100\}$-diamond (Fig. 4). It has been also reported that the $\mathrm{CH}_{2}$ species is promising as a migrating admolecule ${ }^{15}$ on $\{111\}-(1 \times 1)$ : $\mathrm{H}$ surfaces that do not support the dimerization processes. Two-dimensional nucleation on terraces is probably suppressed by plasma etching process even when $x_{\mathrm{s}}<w$. This is particularly true for the plasma-assisted diamond growth using extremely low $\left[\mathrm{CH}_{4}\right] /\left[\mathrm{H}_{2}\right] .{ }^{22,27-30}$

The longer migration length of phosphorus admolecules (cf. hydrocarbon) might be ascribed to a lower plasmaetching rate when compared with hydrocarbon admoleculesconversely, a higher rate in the case of boron admolecules.
However, the specific phosphorus species $\left(\mathrm{PH}, \mathrm{PH}_{2}\right.$, and so forth $^{31}$ ) actually involved in the surface reactions cannot be addressed here. Also, we have not experimentally clarified the conformity between the actual terrace width and the nominal value of $w=d / \tan \theta_{\text {mis }}$ used in Figs. 2(b) and 2(c). Further study will be required to verify the estimated values of $x_{\mathrm{s}}$ and $\tilde{x}_{\mathrm{s}}$ in the present study. Nonetheless, the ratio of $\tilde{x}_{\mathrm{s}} / x_{\mathrm{s}}>1$ should be essential for the enhancement of $[\mathrm{P}] /[\mathrm{C}]$ when using the largest possible $w$ of vicinal substrates.

In summary, we propose that if step-flow growth is predominant on a vicinal substrate face, dopant or impurity concentrations in different materials can be determined by the relative lengths of $w / 2 x_{\mathrm{s}}^{l}$ and $\tilde{x}_{\mathrm{s}} / x_{\mathrm{s}}$. We provide a possible interpretation that the surface migration length of phosphorus admolecules (ca. $4.2 \mathrm{~nm}$ ) is longer than that of hydrocarbon species (ca. $0.5 \mathrm{~nm}$ ), which maximizes the phosphorus donor concentration of diamond with as large a terrace width of a $\{111\}$-substrate as possible. These findings will be of assistance to reassemble and refine modern semiconducting diamond devices that are comprised of bipolar or tripolar ( $p n$ or pin) junctions. ${ }^{1-3}$

See supplementary material for more details.

The authors thank Misa Yoshida for experimental support and Masahiko Ogura and Toshiharu Makino for valuable discussion. This work was partly supported by the Advanced Low Carbon (ALCA) Technology Research and Development Program Foundation, Japan Science and Technology Agency.

${ }^{1}$ D. Takeuchi, T. Makino, H. Kato, M. Ogura, H. Okushi, H. Ohashi, and S. Yamasaki, Jpn. J. Appl. Phys., Part 1 51, 090113 (2012).

${ }^{2}$ S. Koizumi, K. Watanabe, M. Hasegawa, and H. Kanda, Science 292, 1899 (2001)

${ }^{3}$ N. Mizuochi, T. Makino, H. Kato, D. Takeuchi, M. Ogura, H. Okushi, M. Nothaft, P. Neumann, A. Gali, F. Jelezko, J. Wrachtrup, and S. Yamasaki, Nat. Photonics 6, 299 (2012).

${ }^{4}$ S. Koizumi, M. Kamo, Y. Sato, H. Ozaki, and T. Inuzuka, Appl. Phys. Lett. 71, 1065 (1997).

${ }^{5}$ H. Kato, D. Takeuchi, N. Tokuda, H. Umezawa, H. Okushi, and S. Yamasaki, Diamond Relat. Mater. 18, 782 (2009).

${ }^{6}$ T. A. Grotjohn, D. T. Tran, M. K. Yaran, S. N. Demlow, and T. Schuelke, Diamond Relat. Mater. 44, 129 (2014).

${ }^{7}$ H. Kato, H. Umezawa, N. Tokuda, D. Takeuchi, H. Okushi, and S. Yamasaki, Appl. Phys. Lett. 93, 202103 (2008).

${ }^{8}$ Y. Bar-Yam and T. D. Moustakas, Nature 342, 786-787 (1989).

${ }_{9}^{9}$ A. De Vita, G. Galli, A. Canning, and R. Car, Nature 379, 523 (1996).

${ }^{10}$ J. C. Angus and C. C. Hayman, Science 241, 913 (1988).

${ }^{11}$ B. V. Derjaguin and D. V. Fedoseev, Sci. Am. 233, 102 (1975).

${ }^{12}$ B. V. Spitzyn, L. L. Bouilov, and B. V. Derjaguin, J. Cryst. Growth 52, 219 (1981).

${ }^{13}$ M. Frenklach, S. Skokov, and B. Weiner, Nature 372, 535 (1994).

${ }^{14}$ M. Frenklach and S. Skokov, J. Phys. Chem. B 101, 3025 (1997).

${ }^{15}$ K. Larsson and J.-O. Carlsson, Phys. Status Solidi A 186, 319 (2001).

${ }^{16}$ S. J. Harris and D. G. Goodwin, J. Phys. Chem. 97, 23 (1993).

${ }^{17}$ C. C. Battaile, D. J. Srolovitz, I. I. Oleinik, D. G. Pettifor, A. P. Sutton, S. J. Harris, and J. E. Butler, J. Chem. Phys. 111, 4291 (1999).

${ }^{18}$ W. K. Burton, N. Cabrera, and F. C. Frank, Philos. Trans. R. Soc. London, A 243, 299 (1951).

${ }^{19}$ M. Ogura, H. Kato, T. Makino, H. Okushi, and S. Yamasaki, J. Cryst. Growth 317, 60 (2011).

${ }^{20}$ M. Kamo, Y. Sato, S. Matsumoto, and N. Setaka, J. Cryst. Growth 62, 642 (1983).

${ }^{21}$ R. Ohtani, T. Yamamoto, S. D. Janssens, S. Yamasaki, and S. Koizumi, Appl. Phys. Lett. 105, 232106 (2014).

${ }^{22}$ S. Koizumi, T. Teraji, and H. Kanda, Diamond Relat. Mater. 9, 935 (2000). 
${ }^{23}$ S. Koizumi, M. Kamo, Y. Sato, S. Mita, A. Sawabe, A. Reznik, C. UzanSaguy, and R. Kalish, Diamond Relat. Mater. 7, 540 (1998).

${ }^{24}$ M. Nesládek, K. Meykens, K. Haenen, L. M. Stals, T. Teraji, and S. Koizumi, Phys. Rev. B 59, 14852 (1999).

${ }^{25}$ M. Katagiri, J. Isoya, S. Koizumi, and H. Kanda, Appl. Phys. Lett. 85, 6365 (2004).

${ }^{26}$ S. P. Mehandru and A. B. Anderson, J. Mater. Res. 5, 2286 (1990).

${ }^{27}$ H. Watanabe, D. Takeuchi, S. Yamanaka, H. Okushi, K. Kajimura, and T. Sekiguchi, Diamond Relat. Mater. 8, 1272 (1999).
${ }^{28}$ D. Takeuchi, H. Watanabe, S. Yamanaka, H. Okushi, and K. Kajimura, Diamond Relat. Mater. 9, 231 (2000).

${ }^{29}$ N. Tokuda, T. Makino, T. Inokuma, and S. Yamasaki, Jpn. J. Appl. Phys., Part 1 51, 090107 (2012).

${ }^{30}$ M.-A. Pinault-Thaury, B. Berini, I. Stenger, E. Chikoidze, A. Lusson, F. Jomard, J. Chevallier, and J. Barjon, Appl. Phys. Lett. 100, 192109 (2012).

${ }^{31}$ T. Van Regemorter and K. Larsson, J. Phys. Chem. A 112, 5429 (2008). 